\title{
Bioinformatic prediction of the antigenic epitopes of recombinant ferritin of Echinococcus granulosus
}

\author{
XUELEI LIU ${ }^{1,2}$, HUI ZHAO ${ }^{1}$, WENYAN CAO ${ }^{1}$, YUMEI LIU ${ }^{1}$, CHUNTAO ZHANG $^{2}$,

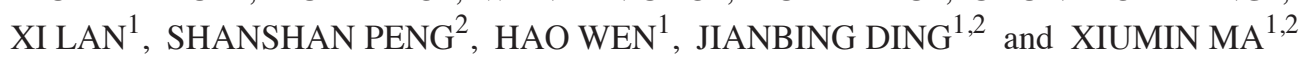 \\ ${ }^{1}$ State Key Laboratory Incubation Base of Xinjiang Major Diseases Research (2010DS890294) and
}

Xinjiang Key Laboratory of Echinococcosis, First Affiliated Hospital of Xinjiang Medical University, Urumqi, Xinjiang 830000;

${ }^{2}$ Department of Immunology, College of Basic Medicine of Xinjiang Medical University, Urumqi, Xinjiang 830011, P.R. China

Received October 29, 2014; Accepted July 21, 2015

DOI: $10.3892 / \mathrm{mmr} .2015 .4575$

\begin{abstract}
Echinococcosis is a zoonotic parasitic disease affecting humans and other mammals, which is mainly caused Echinococcus at larval stages. It is predominantly endemic in Chinese pasture regions, including Xinjiang, Qinghai, Gansu and Ningxia. The aim of the present study was to predict the T- and B-combined epitopes of Echinococcus granulosus (Eg). ferritin, and to analyze its secondary structure using online software. Prediction of the T- and B-combined epitopes of Eg. ferritin was performed using IEDB, SYFPEITHI and LEPS software, which are used to identify common areas of T- and B-cells. The results of the present study identified several potential antigenic epitopes of Eg. ferritin, including seven B-cell antigen epitope amino acid sequences with high values: 8-16, 54-61, 70-75, 80-90, 103-109, 117-124 and 167-173; and four T-cell antigen epitope amino acid sequences with high values: 85-93, 105-113, 133-141 and 157-165. Furthermore, a combined epitope region comprising an 105-109 amino acid sequence was identified. In conclusion, using bioinformatic methods, the present study confirmed the existence of Eg. ferritin on four T-cell antigen epitopes, seven B-cell antigen epitopes, and one T- and B-combined epitope region. These findings provide significant information for further investigation of the antigenicity of Eg. ferritin and the development of highly efficient epitope vaccines.
\end{abstract}

Correspondence to: Dr Xiumin Ma, State Key Laboratory Incubation Base of Xinjiang Major Diseases Research (2010DS890294) and Xinjiang Key Laboratory of Echinococcosis, First Affiliated Hospital of Xinjiang Medical University, 137 Liyushan South Road, Urumqi, Xinjiang 830000, P.R. China

E-mail: maxiumin1210@163.com

Dr Jianbing Ding, Department of Immunology, College of Basic Medicine of Xinjiang Medical University, 393 Xinyi Road, Urumqi, Xinjiang 830011, P.R. China

E-mail: 331044524@qq.com

Key words: Eg. ferritin, antigen epitope, bioinformatics

\section{Introduction}

Echinococcosis, also termed cystic echinococcosis, is a type of zoonotic parasitic disease, which is caused by infection with Echinococcus larvae. Echinococcosis is a severely harmful disease affecting humans and animals, and is associated with high rates of mortality and disability worldwide (1), particularly in developing countries. China is one of the countries with the highest incidence of echinococcosis. Echinococcosis is predominantly endemic in pasture regions, including Xinjiang, Qinghai, Gansu and Ningxia, as well as in semi-pasture regions. At present, surgery is considered the primary therapeutic strategy for the treatment of echinococcosis, whereas drug therapy is supplementary and less efficient. In recent years, vaccination against Echinococcus has attracted increased attention (2).

Ferritin is a multifunctional and multimeric protein, which is widely distributed amongst organisms and has a significant role in the regulation of immune function (3-6). Previous studies have identified Echinococcus granulosus (Eg.) ferritin as an antigenic molecule, which is associated with a certain immunological protection. In the 1990s, Eresfeld and Craig (7) cloned the Eg. ferritin gene, and determined that it exhibits immunogenicity and can be used to diagnose echinococcosis. Therefore, the Eg. ferritin gene has attracted increasing attention. The present study aimed to predict the T-cell and B-cell antigen epitopes of Eg. ferritin and perform sequence analysis, in order to diagnose and treat hepatic echinococcosis. The present study may provide novel evidence supporting the development of an epitope vaccine against echinococcosis.

\section{Materials and methods}

Primary reagents. TRIzol ${ }^{\circledR}$, Taq enzyme and an AMV First Strand cDNA Synthesis kit were purchased from Invitrogen Life Technologies (Carlsbad, CA, USA). The DL2000 DNA Marker was purchased from Takara Biotechnology Co., Ltd. (Dalian, China).

Specimen collection of Echinococcus granulosus. Fresh livers of sheep which had naturally contracted Echinococcus granulosus, as identified by vesicae in the liver, were obtained from Xinjiang Slaughterhouse (Urumqi, China) and cystic fluid was extracted 
using a $50 \mathrm{ml}$ sterile syringe. The fluid was transferred into a centrifuge tube, and the protoscolex were allowed to naturally precipitate. Following rinsing three times with sterile saline, the protoscolex were collected and stored at $4{ }^{\circ} \mathrm{C}$ for further analysis. The study was approved by the ethics committee (ZACUS20130425002) of Xinjiang Medical University (Urumqi, China).

Primer design and synthesis. According to the Eg. ferritin gene sequence (GenBank ID: Z31712; http://www.ncbi.nlm. nih.gov/nuccore/Z31712), the following primer was designed using DNAman software (LynnonBiosoft Corp., San Ramon, CA, USA): Eg. ferritin, forward 5'-CGGAATTCATGAGGA ATGCGAACGTG-3' and reverse 5'-CGCAAGCTTTGATAA AAAATTATTTGT-3'. The primer was synthesized by Sangon Biotech Co., Ltd. (Shanghai, China).

Analytical software. The Self-Optimized Prediction Method with Alignment (SOPMA) server (http://npsa-pbil.ibcp. fr/cgi-bin/npsa_automat.pl?page=/NPSA/npsa_sopma.\%20.ht $\mathrm{ml}$ ) was used to predict the secondary structure of Eg. ferritin; the Immune Epitope Database(IEDB; http://tools.immuneepitope.org/tools/bcell/iedb_input) and Linear Epitope Prediction Based on Propensity Scale and SVM (LEPS; http://leps. cs.ntou.edu.tw/index.php) tool were used to predict the B-cell epitope; and the SYFPEITHI (http://www.syfpeithi.de) database and IEDB tools were used to predict the T-cell epitope. The online software, 3D Ligandsite (http://www.sbg.bio.ic.ac. uk/ 3dligandsite/) and RasMol (http://www.rasmol.org/) were used to predict the three dimensional (3D) structure of Eg. ferritin.

Extraction of total RNA from Echinococcus granulosus protoscolex and synthesis of cDNA. Echinococcus protoscolices were ground between three and six times in liquid nitrogen (provided by The State Key Laboratory Incubation Base of Xinjiang Major Diseases Research, The First Affiliated Hospital of Xinjiang Medical University, Urumqi, China) prior to RNA extraction in a sterile mortar. A total of $1 \mathrm{ml} \mathrm{TRIzol}{ }^{\circledR}$ (Invitrogen Life Technologies, Inc.) was then added per $100 \mathrm{ml}$ sample, and ground between three and six times. Total RNA was extracted using TRIzol ${ }^{\circledR}$ according to the manufacturer's instructions. The RNA concentration was determined using an ultraviolet spectrophotometer (ND1000; NanoDrop; Thermo Fisher Scientific, Waltham, MA, USA) and was dissolved in $50 \mu$ l water treated with diethylpyrocarbonate (Tianjing Fuyu Chemical Co., Ltd., Tianjing, China). The samples $(5 \mu \mathrm{l})$ were then run on a $1.2 \%$ 3-(N-morpholino) propanesulfonic acid (MOPS)-formaldehyde denaturing gel (Tianjing Fuyu Chemical Co., Ltd). RNA was reverse-transcribed into cDNA using a RevertAid ${ }^{\mathrm{TM}}$ First strand cDNA Synthesis kit (Thermo Fisher Scientific) according to the manufacturer's instructions. Reactions were performed using $2 \mu \mathrm{l}$ cDNA in a $20-\mu 1$ reaction volume and the following thermocycling profile: $10 \mathrm{~min}$ of denaturation at $95^{\circ} \mathrm{C}, 40$ cycles of denaturation at $95^{\circ} \mathrm{C}$ for $15 \mathrm{sec}$ and $60 \mathrm{sec}$ of extension at $60^{\circ} \mathrm{C}$.

Cloning andidentification of the Eg.ferritingene. The Eg. ferritin gene was cloned from the protoscolex cDNA. Amplification of Eg95 was performed in a $20-\mu 1$ mixture containing $1 \mu \mathrm{l}$ cDNA template, $2 \mu 110 \mathrm{X}$ buffer, $0.5 \mu \mathrm{l}$ of each primer, $0.5 \mu 110 \mathrm{~mm}$ dNTP, $0.5 \mu 1$ Taq enzyme and $15.5 \mu \mathrm{l}$ pure water (2XTaq PCR
Master Mix or Maxima SYBR Green/ROX qPCR Master Mix; Invitrogen Life Technologies, Inc.). The cycling conditions of the polymerase chain reaction (PCR) were as follows: Initial denaturation at $95^{\circ} \mathrm{C}$ for $6 \mathrm{~min}$, followed by 30 consecutive cycles of denaturation at $95^{\circ} \mathrm{C}$ for $30 \mathrm{sec}$, annealing at $55^{\circ} \mathrm{C}$ for $30 \mathrm{sec}$ and extension at $72^{\circ} \mathrm{C}$ for $1 \mathrm{~min}$, and a final extension step at $72^{\circ} \mathrm{C}$ for $5 \mathrm{~min}$. The PCR products were subsequently detected using 1.2\% agarose gel electrophoresis (Sigma-Aldrich, St. Louis, MO, USA).

Amino acid sequences coded by Eg. ferritin. Validation of gene sequence analysis was performed and the corresponding amino acid sequences were identified using the DNAman software program. Multiple sequences were detected, according to the $\mathrm{Eg}$. ferritin gene sequence obtained from GenBank.

Prediction of secondary protein structure. Prediction of the secondary structure of the Eg. ferritin protein was performed using the online SOPMA server (http://npsa-pbil.ibcp. fr/cgi-bin/npsa_automat.pl?page=/NPSA/npsa_sopma.\%20.ht $\mathrm{ml})$.

B cell epitope prediction software. Predictions of B cell hydrophilicity, antigenicity and flexibility were made using the IEDB (http://tools.immuneepitope.org/tools/bcell/iedb_input) and LEPS (http://leps.cs.ntou.edu.tw/index.php) online prediction software.

T-cell epitope prediction software. Prediction of the potential major histocompatibility complex (MHC)-I type human leukocyte antigen (HLA)-A 0201 restrictive T-cell epitope was made using the SYFPEITHI (http://www.syfpeithi.de) and IEDB (http://tools.immuneepitope.org/tools/bcell/iedb_input) online resources.

Prediction of the tertiary structure of Eg. ferritin. The tertiary structure of Eg. ferritin was predicted using the 3DLigandsite (http://www.sbg.bio.ic.ac.uk/ 3dligandsite/) online server, combined with RasMol software, in order to analyze and determine different models of presentation, including Cartoon, Structure and Group.

\section{Results}

Extraction of total RNA from Echinococcus granulosus protoscolex. The absorption value of the extracted total RNA was detected at 260 and $280 \mathrm{~nm}$ wavelengths, using a nucleic acid analyzer. The value of protoscolex RNA was 1.94; demonstrating that the RNA was extracted successfully. The resulting MOPS-formaldehyde denaturing gel electrophoresis of Eg. ferritin is shown in Fig. 1.

Cloning of the Eg. ferritin gene. cDNA was subsequently used as a template for PCR amplification using an Eg. ferritin primer. The PCR products were verified using 1.2\% agarose gel electrophoresis. As shown in Fig. 2. the Eg. ferritin PCR products resulted in a specific band at $653 \mathrm{bp}$, whereas no such band was detected in the negative control group, in which water was used instead of template. This indicated that a specific PCR fragment had been successfully amplified from the cDNA. 
Table I. Predicted major histocompatibility complex-I type human leukocyte antigen-A 0201 restrictive T-cell epitopes using SYFPEITHI and IEDB.

\begin{tabular}{|c|c|c|c|}
\hline Order & Initiation site & $\begin{array}{c}\text { Amino } \\
\text { acid sequence }\end{array}$ & Score \\
\hline \multicolumn{4}{|c|}{ SYFPEITHI } \\
\hline 1 & 143 & KLAGYVTNL & 30 \\
\hline 2 & 133 & FLGEQVSDI & 25 \\
\hline 3 & 92 & GLEAMEML & 21 \\
\hline 4 & 150 & NLKRCGPGL & 21 \\
\hline 5 & 105 & EVNESLLAL & 20 \\
\hline 6 & 23 & ELYASYLYL & 19 \\
\hline 7 & 109 & SLLALRGVA & 19 \\
\hline 8 & 21 & NMELYASYL & 18 \\
\hline 9 & 98 & MALKIEREV & 18 \\
\hline 10 & 157 & GLGEYIFDK & 18 \\
\hline 11 & 110 & LLALRGVAN & 17 \\
\hline 12 & 112 & ALRGVANKN & 17 \\
\hline 13 & 140 & DIKKLAGYV & 17 \\
\hline 14 & 85 & QTTEWASGL & 16 \\
\hline 15 & 102 & IEREVNESL & 16 \\
\hline \multicolumn{4}{|l|}{ IEDB } \\
\hline 1 & 9 & HEECERGIN & 100 \\
\hline 2 & 11 & ECERGINRQ & 100 \\
\hline 3 & 13 & ERGINRQIN & 100 \\
\hline 4 & 56 & EEEREHAIK & 100 \\
\hline 5 & 164 & DKETLQGGE & 99 \\
\hline 6 & 158 & LGEYIFDKE & 96 \\
\hline 7 & 52 & AKASEEERE & 95 \\
\hline 8 & 60 & EHAIKLMRY & 94 \\
\hline 9 & 70 & CGRGGRIVY & 94 \\
\hline 10 & 134 & LGEQVSDIK & 94 \\
\hline 11 & 58 & EREHAIKLM & 93 \\
\hline 12 & 113 & LRGVANKNN & 93 \\
\hline 13 & 103 & EREVNESLL & 91 \\
\hline 14 & 86 & TTEWASGLE & 90 \\
\hline 15 & 40 & DDVALPGFR & 90 \\
\hline
\end{tabular}

IEDB, Immune Epitope Database.

Amino acid sequence coded by Eg. ferritin. The corresponding amino acid sequence to be translated from the Eg. ferritin gene was predicted using DNAman online software. The following 176 amino acid residue was identified: MSLVRQNFHEECERGINRQIN MELYASYLYLAMSQHFDRDDVALPGFREFFAKASEEE REHAIKLMRYQCGRGGRIVYQDIAKPQTTEWASGLEA MEMALKIEREVNESLLALRGVANKNNDSQFCEFLEGE FLGEQVSDIKKLAGYVTNLKRCGPGLGEYIFDKETLQ GGEK.

Prediction of the secondary structure of Eg. ferritin antigenic protein. Prediction of the secondary structure of Eg. ferritin antigenic protein was made using the online
Table II. T- and B-combined epitope.

\begin{tabular}{lcc}
\hline Epitope & Predicted region & $\begin{array}{c}\text { Amino acid } \\
\text { sequence }\end{array}$ \\
\hline B-cell & $103-109$ & ER $\underline{E V N E S}$ \\
T-cell & $105-113$ & $\underline{E V N E S L L A L}$ \\
B- and T-combined & $105-109$ & $\underline{E V N E S}$ \\
\hline
\end{tabular}

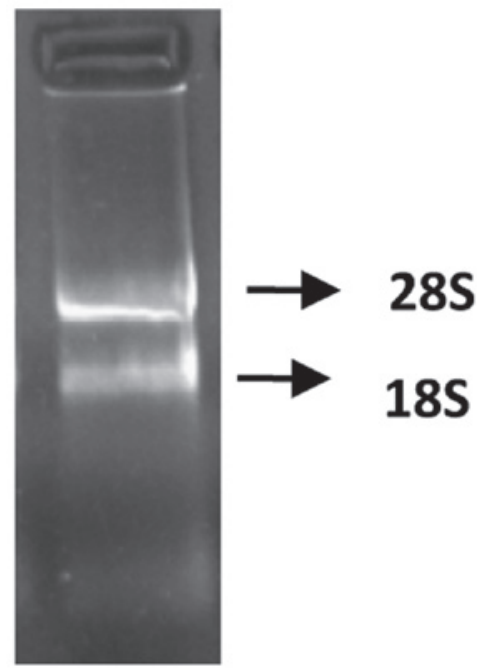

Figure 1. Results of Echinococcus granulosus ferritin gel electrophoresis.

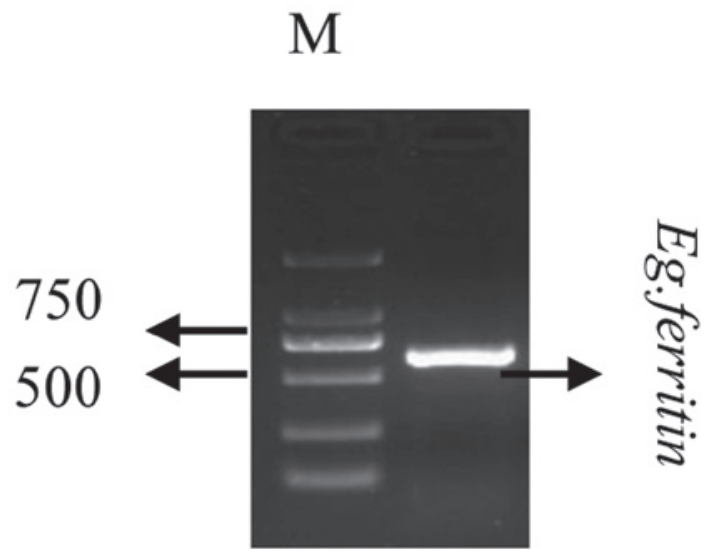

Figure 2. Agarose gel electrophoresis of polymerase chain reaction products of Echinococcus granulosus (Eg.) ferritin (right-hand lane). M, marker.

software, SOPMA. $\alpha$-helix structures accounted for $73.41 \%$ of the total amino acid sequence, and $\beta$-fold structures and random coils accounted for 4.05 and $16.76 \%$ of the total amino acid sequence, respectively. The distribution of the different structures of Eg. ferritin antigenic protein is shown in Fig. 3.

B-cell antigen epitope prediction of Eg. ferritin. A prediction was made on the combined hydrophilicity, antigenicity and flexibility of Eg. ferritin using IEDB and LEPS (http://leps.cs.ntou. edu.tw/index.php) online software. Regions with high values 

$\begin{array}{ccccccr}10 & 20 & 30 & 40 & 50 & 60 & 70 \\ 1 & 1 & 1 & 1 & 1 & 1 & 1 \\ \text { MSLVRQNFHEECERGINRQINMELYASYLYLAMSQHFDRDDVALPGFREFFAKASEEEREHAIKLMRYQC }\end{array}$

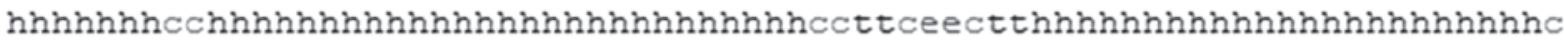
GRGGRIVYQDIAKPQTTEWASGLEAMEMALKIEREVNESLLALRGVANKNNDSQFCEFLEGEFLGEQVSD

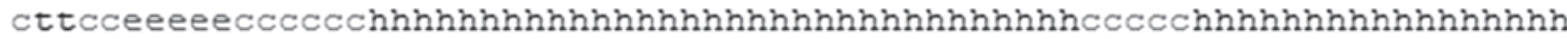
IKKLAGYVTNLKRCGPGLGEYIFDKETLQGGEK

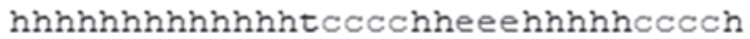

$\begin{array}{lllrr}\text { SOPMA : } & & & \\ \text { Alpha helix } & (\mathrm{Hh}): & 127 \text { is } & 73.41 \% \\ 3_{10} \text { helix } & (\mathrm{Gg}): & 0 \text { is } & 0.00 \% \\ \text { Pi helix } & (\mathrm{Ii}): & 0 \text { is } & 0.00 \% \\ \text { Beta bridge } & (\mathrm{Bb}): & 0 \text { is } & 0.00 \% \\ \text { Extended strand } & (\mathrm{Ee}): & 10 \text { is } & 5.78 \% \\ \text { Beta turn } & (\mathrm{T}): & 7 \text { is } & 4.05 \% \\ \text { Bend region } & (\mathrm{Ss}): & 0 \text { is } & 0.00 \% \\ \text { Random coil } & (\mathrm{Cc}): & 29 \text { is } & 16.76 \% \\ \text { Ambigous states } & (?): & 0 \text { is } & 0.00 \% \\ \text { Other states } & & : & 0 \text { is } & 0.00 \%\end{array}$

Figure 3. Secondary structure of Echinococcus granulosus ferritin.

\section{A}

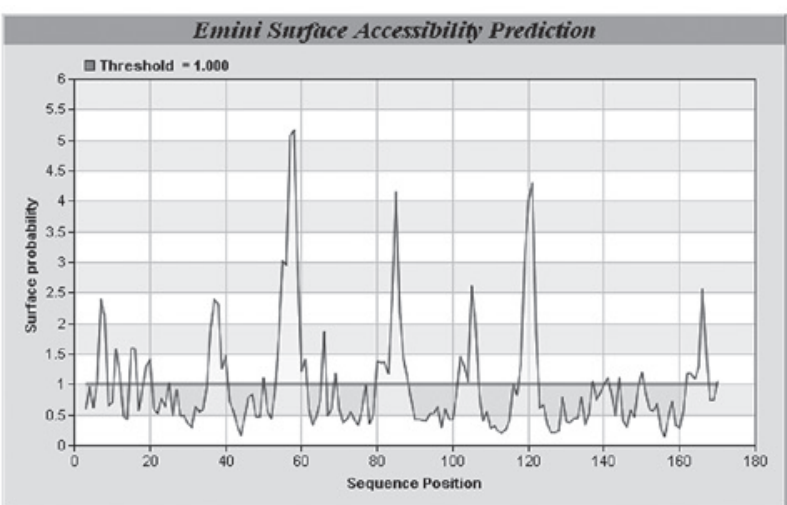

$\mathbf{C}_{1}$

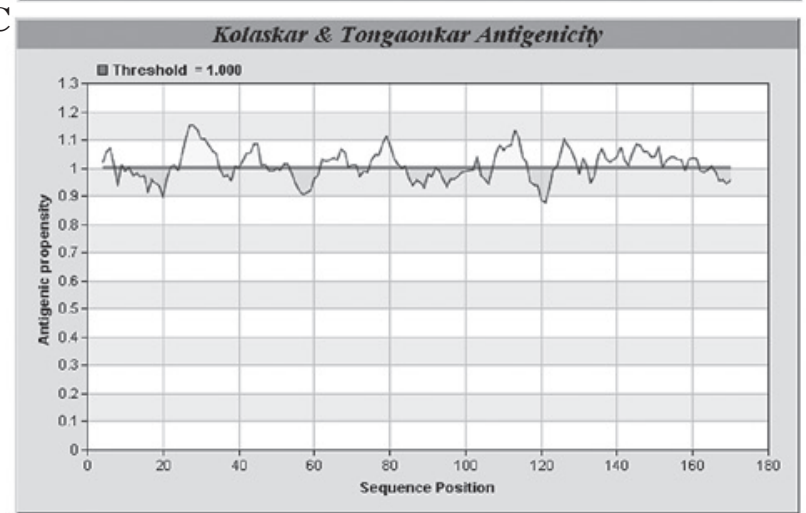

$\mathbf{E}$

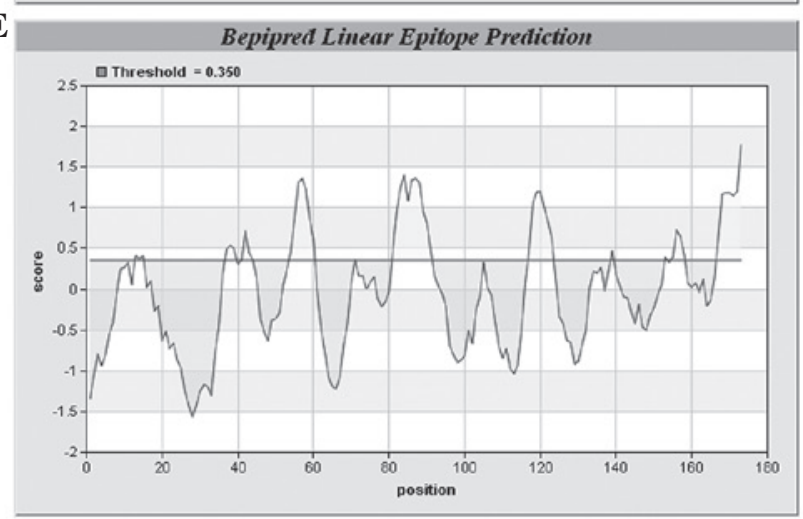

B

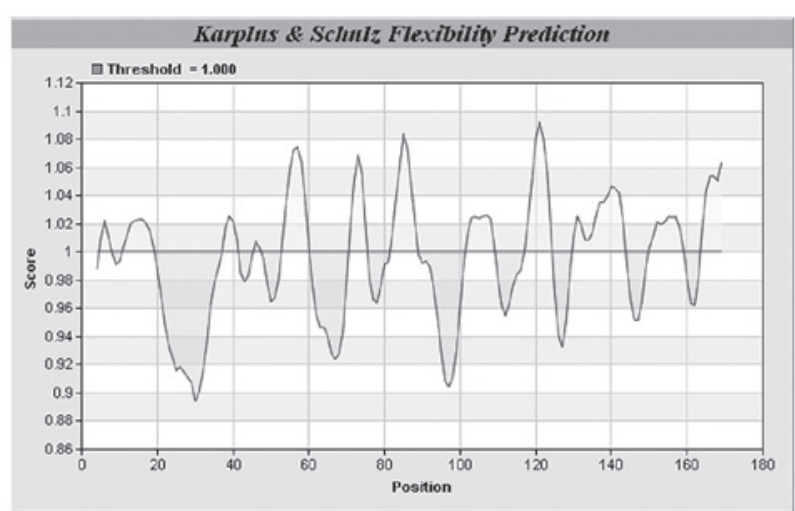

D

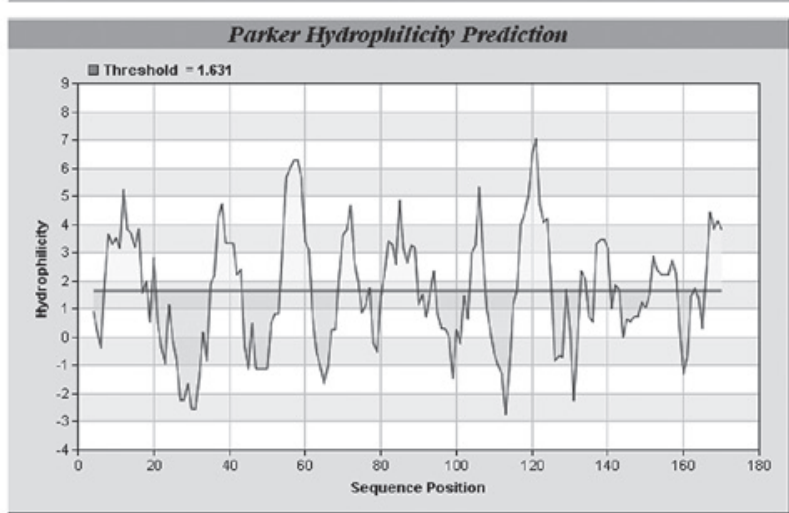

F

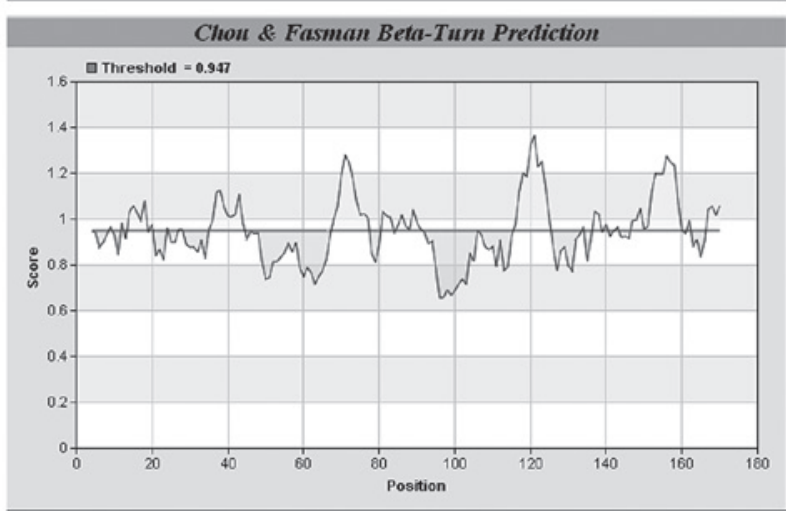

Figure 4. Online B-cell epitope prediction with the Immune Epitope Database, based on several parameters. (A) Prediction of surface accessibility. (B) Prediction of flexibility of the region. (C) Prediction of antigenicity. (D) Prediction of hydrophilicity. (E) Prediction of linear epitope. (F) Prediction of $\beta$ angle. 

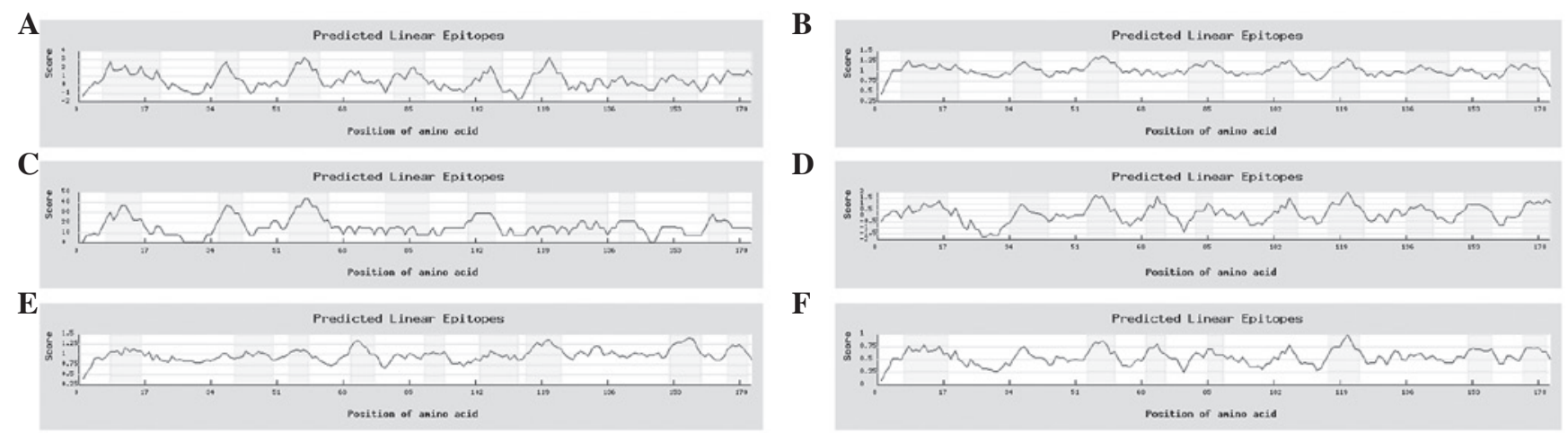

Figure 5. Linear Epitope Prediction Based on Propensity Scale and SVM B-cell epitope prediction, based on several parameters. (A) Prediction of the hydrophilcity, (B) surface accessibility, (C) polarity, (D) flexibility of the region, (E) $\beta$ angle, and (F) antigenicity.

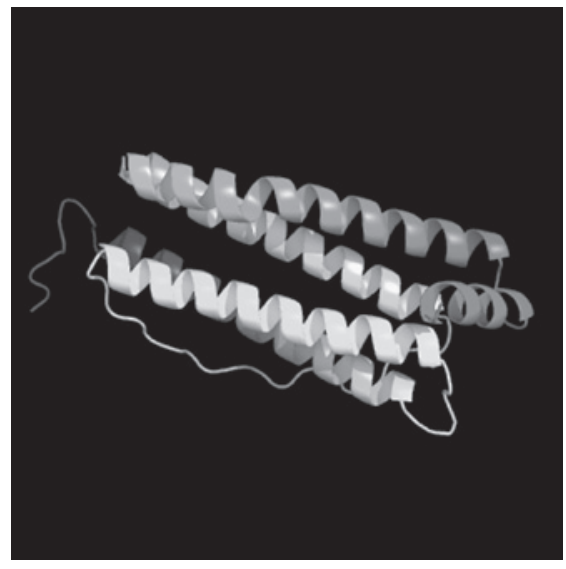

Figure 6. Tertiary structure simulation model of Echinococcus granulosu ferritin, analyzed using 3DLigandsite.

are considered to be potential B-antigen epitopes. According to the predicted results, several high value amino acid sequences were identified (Figs. 4 and 5). Combining the results of the two software analyses, seven potential B-antigen epitopes were identified, comprising the 8-16, 54-61, 70-75, 80-90, 103-109, 117-124 and 167-173 amino acid sequence regions.

T-cell antigen epitope prediction of Eg. ferritin. In order to obtain the most accurate results, the SYFPEITHI (http://www. syfpeithi.de) database and IEDB (http://tools.immuneepitope. org/tools/bcell/iedb_input) online prediction tool were used to predict the MHC-I type HLA-A 0201 restrictive T-antigen epitope. These each identified 15 regions with high values. The results of the analyses are shown in Table I. Combining the results of the two software analyses, four potential T-cell antigen epitopes, including the 85-93, 105-113, 133-141 and 157-165 amino acid sequence regions, were identified.

Using multiple sequence alignment with DNAman software and comparing the potential T-cell and B-cell epitopes, the highly overlapped amino acid sequence 105-109 was predicted as a T- and B-combined epitope (Table II).

Analyses of Eg. ferritin tertiary structure. Segments of the Eg. ferritin amino acid code were submitted to the 3DLigandsite server (http://www.sbg.bio.ic.ac.uk/ 3dligandsite/), in order to predict and analyze the 3D structure of the protein (Fig. 6).
A

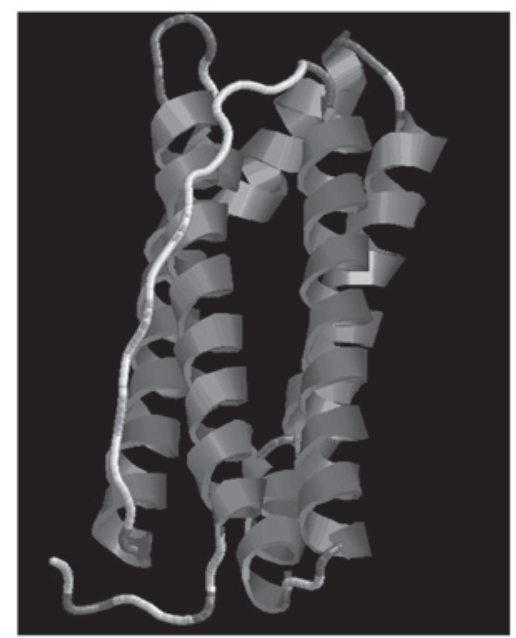

B

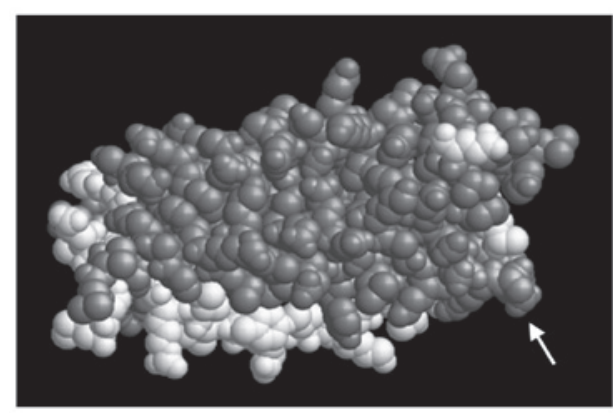

C

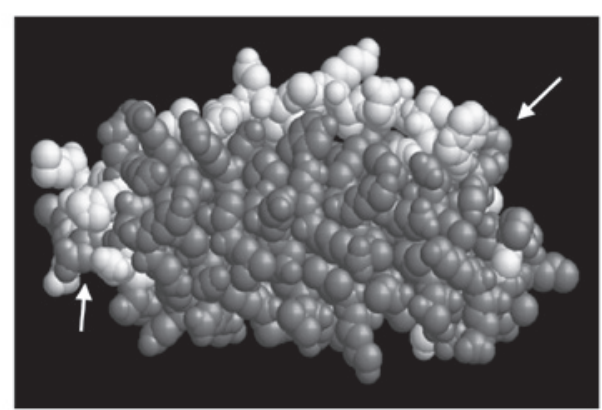

Figure 7. Different model demonstrations of the tertiary structure of Echinococcus granulosus ferritin. (A) Structural model. (B) Anterior view of the group structure. (C) Posterior view of the group structure. Arrows indicate locations which may form $\mathrm{T}$ or $\mathrm{B}$ cell epitopes.

Different demonstration models were applied, including the Structure and Group (Fig. 7A-C), to determine the specific 
position of each amino acid on the tertiary structure of Eg. ferritin using RasMol and 3D Ligandsite analysis software. A marked similarity was observed between an area in the structural model and the flexible area predicted by the secondary structure analysis. The Group structure model detected that this assembled area and was distributed at the surface of the structure, indicating that it is most likely the combined epitope of antigen and antibody.

\section{Discussion}

China remains one of the countries with a high incidence of echinococcosis, which is a significant factor affecting economic development and public health in western China. Therefore, the identification of an antigen with high specificity and high sensitivity is important for the diagnosis and treatment of echinococcosis. It has previously been demonstrated that Eg. ferritin attains a protective immunity of $85.6 \%$ in animals; therefore, it can be considered as a potential antigen for investigation $(8,9)$.

Early investigation by Schuler (10) identified the FMDV immune locus, which is an antigenic epitope and specific chemical group among antigen molecules for determining antigenic specificity, also termed an antigen determinant. Epitopes can be divided into B-cell epitopes and T-cell epitopes. B-cell epitopes are located on the surface of the antigenic molecule and induce the humoral immune response and production of specific antibodies from B-cells. T-cell epitopes are linear peptides, which, following the antigen-presenting cell process, delivers antigens from the MHC molecule to the T-cell receptor and is connected to the cellular immune response (11). Epitope vaccines are produced according to epitope amino acid sequences (12) and epitope vaccines have become an important focus of molecular vaccine investigations; Kouguchi et al (13) demonstrated that the EmY162 recombinant antigen can induce $74.3 \%$ immune protection in mice. The most important process of epitope vaccine production is the identification of a sequence with a highly specific epitope location (14).

In the prediction of the secondary protein structure, random coils and $\beta$-folds are considered the prominent structural features, the majority of which appear predominantly on the surface of the protein antigens and are beneficial for the recognition of antigens, and are therefore likely to be an antigenic epitope (15). The present study demonstrated that random coils accounted for $16.76 \%$ and $\beta$-folds accounted for $4.05 \%$ of the total antigen protein structure of Eg. ferritin. These results indicated that these structures are within the distribution region of the antigenic epitope with marked immunogenicity $(16,17)$. The higher the level flexibility, the easier it is to form an antigenic epitope. Antigen accessibility is made possible through contact between amino acid residues and solvent molecules, indicating the distribution of internal and external antigen residues. In the 3D protein structure, globular or oval structures, which are formed by peptide coils and folds, always form a hydrophilic molecule and a hydrophobic nuclear molecule, enabling stability of the 3D structure due to the presence of hydrophobic and hydrogen bonds (11). With prediction of the flexibility of the antigen protein and epitope accessibility in the present study, further evidence were gained to confirm this.
In the T-cell epitope prediction in the present study, an MHC-I type antigenic epitope was predicted with high accuracy. HLA-A 0201 restrictive T-cells are most common among the Han Chinese population $(11,18,19)$. In the present study, nine peptides of HLA-A0201 MHC-I type antigenic epitopes were predicted using online software, and four regions with high values were identified: $85-93,105-113,133-141$ and 157-165. The present study also identified a potential T- and B-combined epitope, which possessed a highly overlapped region (105-109). These results may provide evidence supporting the production of a $\mathrm{T}$ - and $\mathrm{B}$-antigen epitope vaccine, contributing to the therapy of echinococcosis in terms of humoral and cellular immunity.

Eg. ferritin has the potential to form T-cell and B-cell epitopes $(20,21)$. The present study used IEDB and LEPS online software to predict the potential B-cell epitope of Eg. ferritin. In total, seven amino acid sequence positions were identified (8-16, 54-61, 70-75, 80-90, 103-109, 117-124 and 167-173), which readily form B-cell epitopes. Furthermore, the present study also predicted the T-cell epitope using the SYFPEITHI and IEDB online servers. This identified four amino acid sequences (85-93, 105-113, 133-141 and 157-165), which readily form T-cell epitopes. Following observation of the potential T-cell and B-cell epitopes, a highly overlapped sequence was found (105-109). In conclusion, the results of the present study provide evidence supporting the production of a highly efficient and safer epitope vaccine, and establishes the foundation for the treatment of echinococcosis.

\section{Acknowledgements}

The present study was supported by the Scientific Research Project of Science Department of Xinjiang Autonomous Region (grant no. 2012211A034), and the National Natural Science Foundation (grant nos. 31160194, 81260253,30960358, 81160378; 31000411, 30901374, 81060135 and 30860263).

\section{References}

1. No authors listed: New influenza A (HINl) virus: Global epidemiological situation, June 2009. Wkly Epidemiol Rec 84: 249-257, 2009.

2. Wen H and Ding Z: Atlas of Echinococcosis. 2nd edition. Science Press, Shanghai, p9, 2008 (In Chinese).

3. Ponka P: Recent advances in cellular iron metabolism. J Trace Elem Exp Med 16: 201-217, 2003.

4. Wang QL, Kong B and Huang HQ: Progress in structural and functional study of nanometer protein shell of the ferritin. Prog Chem 16: 516-519, 2004 (In Chinese).

5. Qadri F, Jonson G, Begum YA, Wennerås C, Albert MJ, Salam MA and Svennerholm AM: Immune response to the mannose-sensitive hemagglutinin in patients with cholera due to Vibrio cholerae O1 and O139. Clin Diagn Lab Immunol 4: 429-434, 1997.

6. Qadri F, Ryan ET, Faruque AS, Ahmed F, Khan AI, Islam MM, Akramuzzaman SM, Sack DA and Calderwood SB: Antigen-speeific immunoglobulin A antibodies secreted from circulating B cells are an effective marker for recent local immune responses in patients with cholera: Comparison to antibody-secreting cell responses and other immunological markers. Infect Immun 71: 4808-4814, 2003.

7. Eresfeld K and Craig PS: Cloning and immunological characterization of Echinococcus granulosus ferritin. Parasitol Res 81: 382-387, 1995.

8. Wang YN, Li ZJ, Li ZY, Bo Y and Zhao W: Recombinant ferritin protects mice against challenge with Echinococcus granulosus. Acta Parasitologica 54: 335-340, 2009. 
9. Wang YN, Ding SQ, Wang J, Zhang Y, Wang J, Wang S, Zhao W: High level expression and identification of recombinant ferritin of Echinococcus granolosus. Chinese Journal of Zoonoses 22: 399-406, 2006

10. Schuler MM, Nastke MD and Stevanovikć S: SYFPEITHI: Database for searching and T-cell epitope prediction. Methods Mol Biol 409: 75-93, 2007.

11. Ma X,Zhou X,Zhu Y, Li Y, Wang H, Mamuti W, Li Y, Wen H and Ding J: The prediction of T- and B-combined epitope and tertiary structure of the Eg95 antigen of Echinococcus granulosus. Exp Ther Med 6: 657-662, 2013.

12. Ben-Yedidia T and Arnon R: Towards an epitope-based human vaccine for influenza. Hum Vaccin 1: 95-101, 2005.

13. Kouguchi H, Matsumoto J, Katoh Y, Oku Y, Suzuki T and Yagi K: The vaccination potential of EMY162 antigen against Echinococcus multilocularis infection. Biochem Biophys Res Commun 363: 915-920, 2007.

14. You L, Brusic V, Gallagher M and Bodén M: Using Gaussian process with test rejection to detect T-Cell epitopes in pathogen genomes. IEEE/ACM Trans Comput Biol Bioinform 7: 741-751, 2010.

15. Xianfei LIU, Hongying Wang, Xiaotao ZHOU, et al: Cloning and bioinformatics prediction of EM18 in Echinococcus multilocularis [J].Chin J Zoonoses 29: 23-26, 2013.
16. Sikic K, Tomic S and Carugo O: Systematic comparison of crystal and NMR protein structures deposited in the protein data bank. Open Biochem J 4: 83-95, 2010.

17. Sy SM, Chen J and Huen MS: The 53BP1-EXPAND1 connection in chromatin structure regulation. Nucleus 1: 472-474, 2010

18. Yan C, Wang R, Li J, Deng Y, Wu D, Zhang H, Zhang H, Wang L, Zhang C, Sun H, et al: HLA-A gene polymorphism defined by high-resolution sequence-based typing in 161 Northern Chinese Han people. Genomics Proteomics Bioinformatics 1: 304-309, 2003.

19. Lin L, Tan B, Pantapalangkoor P, Ho T, Hujer AM, Taracila MA, Bonomo RA and Spellberg B: Acinetobacter baumannii rOmpA vaccine dose alters immune polarization and immunodominant epitopes. Vaccine 31: 313-318, 2013

20. Jonson G, Holmgren J and Svennerholm AM: Identification of a mannose-binding pilus on Vibrio cholerae EI Tor. Microb Pathog 11: 433-441, 1991.

21. Jonson G, Lebens M and Holmgren J: Cloning and sequencing of Vibrio cholerae mannose-sensitive haemagglutinin pilin gene: Localization of mshA within a cluster of type 4 pilin genes. Mol Microbiol 13: 109-118, 1994. 